\title{
Pyogenic thigh abscess caused by Streptococcus constellatus subsp. constellatus in a patient with exacerbation of bronchiectasis: a case report
}

\author{
Yinhe Feng $^{1,2 \# \wedge}$, Yubin Wang ${ }^{1 \# \wedge}$, Chunfang Zeng ${ }^{2} \wedge$, Hui Mao $^{1 \wedge}$ \\ ${ }^{1}$ Department of Respiratory and Critical Care Medicine, West China Hospital, Sichuan University, Chengdu, China; ${ }^{2}$ Department of Respiratory \\ and Critical Care Medicine, People's Hospital of Deyang City, Affiliated Hospital of Chengdu College of Medicine, Deyang, China \\ \#These authors contributed equally to this work. \\ Correspondence to: Hui Mao, MD. Department of Respiratory and Critical Care Medicine, West China Hospital, Sichuan University, No. 37 Guoxue \\ Alley, Chengdu 610041, China. Email: merrymh@126.com.
}

\begin{abstract}
Streptococcus constellatus (S. constellatus) is a Gram-positive commensal bacterium that is commonly found in the oral, nasal, pharyngeal, gastrointestinal, and urogenital tracts. It can be further consisted by three subspecies: subsp, constellatus, subsp. pharynges, and subsp. viborgensis. As an opportunistic pathogen, S. constellatus can cause abscesses and bacteremia, so infection requires timely and accurate identification in clinical practice. There are a few case reports describing the range of infections caused by S. constellatus, which include intracardiac, thoracic, intracranial, and abdominal infections. Here we report the first case of thigh abscess caused by $S$. constellatus subsp. constellatus which was rarely insensitive to penicillin in a patient with exacerbation of bronchiectasis. The patient improved significantly after receiving antibiotic therapy with ceftriaxone and vancomycin, as well as percutaneous catheter drainage guided by color ultrasonography. The thigh abscess did not recur during follow-up. This case report demonstrates that although $S$. constellatus is a rare infectious pathogen, it is important to gain a better understanding of the range of possible infections to ensure timely diagnosis. Furthermore, although the prognosis of most patients with such infections is relatively good, the timely identify the resistant strains and administration of sensitive antibiotics along with abscess drainage may ensure effective treatment.
\end{abstract}

Keywords: Streptococcus constellatus; thigh abscess; bronchiectasis; case report

Submitted Mar 29, 2021. Accepted for publication Jun 11, 2021.

doi: 10.21037/apm-21-740

View this article at: https://dx.doi.org/10.21037/apm-21-740

\section{Introduction}

The Streptococcus anginosus (S. anginosus) group, also known as $S$. milleri group, comprises three Gram-positive commensal species: S. constellatus, S. anginosus, and $S$. intermedius (1). These bacteria are typically distributed in the oral, nasal, pharyngeal, gastrointestinal and urogenital tracts, and could cause less infections than other well-known streptococci (e.g., the group A streptococci and pneumococci).
Thereinto, S. constellatus can be further consisted by three subspecies: subsp, constellatus, subsp. pharynges, and subsp. viborgensis (2). S. constellatus used to be considered opportunistic pathogen and could cause infections in patients with chronic respiratory diseases, cardiovascular diseases, or immunocompromised and immunodeficient diseases (3). Recent studies, however, have detected $S$. constellatus in individuals with normal immunity, often together with certain anaerobic strains, which exacerbate

\footnotetext{
^ ORCID: Yinhe Feng, 0000-0001-8283-214X; Yubin Wang, 0000-0002-3636-9374; Chunfang Zeng, 0000-0002-8403-7454; Hui Mao, 0000-0001-5288-0271.
} 
inflammation $(4,5)$.

S. constellatus infection is not common, and it can manifest as abscesses and bacteremia. S. constellatus was first isolated from an oral abscess in a patient with odontogenic infection, and gradually there are a few case reports describing the range of infections caused by $S$. constellatus, including intracardiac, thoracic, intracranial and abdominal infections (6-9). We are aware of one report of purulent thigh infection caused by Eubacterium lentum mixed with $S$. constellatus in a patient with periodontal disease, but the $S$. constellatus subspecies was not identified (10). Here we report the first case of pyogenic thigh abscess caused by $S$. constellatus subsp. constellatus in a patient with exacerbation of bronchiectasis. This report will improve our understanding of the range of infections associated with $S$. constellatus and perhaps aid in their timely diagnosis. The following case is presented in accordance with the CARE reporting checklist (available at https://apm.amegroups. com/article/view/10.21037/apm-21-740/rc).

\section{Case presentation}

A 64-year-old Chinese man, who was a farmer and had previously been diagnosed with bronchiectasis in a local hospital based on chronic cough and expectoration (lasting $>10$ years), was admitted to our hospital after experiencing exacerbation involving nine days of rigor, moderate fever, increased cough and expectoration of white-yellow purulent sputum, as well as four days of pain in the right thigh. The patient reported no hemoptysis, chest pain, hot flashes, night sweats, obvious dyspnea, or weight loss. Prior to admission to our hospital, he had received levofloxacin $(0.5 \mathrm{~g}$ once a day) for three days at another hospital, but this had no effect on his respiratory symptoms, fever, or thigh pain. He reported having received $\beta$-lactam antibiotics such as penicillins to treat previous exacerbations of bronchiectasis, but he was unable to provide further details about the cause of his bronchiectasis or results of microbiological tests. About three weeks prior to admission to our hospital, he had been diagnosed with gingivitis based on bleeding gums in a clinic, which had resolved after chlorhexidine gargle. He was taking amlodipine besylate $(5 \mathrm{mg})$ and enalapril maleate $(10 \mathrm{mg})$ once daily 5 years for essential arterial hypertension. He reported having quit smoking 10 years prior to admission to our hospital, before which he had been smoking at least 20 cigarettes per day for 30 years. He denied recent travel, surgery or trauma, and he reported no history of infectious diseases, diabetes mellitus or use of alcohol or drugs.

At admission, the patient had a high body temperature $\left(38.5^{\circ} \mathrm{C}\right)$, an increased respiratory rate $(23$ breaths $/ \mathrm{min})$, a low blood pressure $(92 / 61 \mathrm{mmHg})$, a normal heart rate (89 beats/min), and a normal oxygen saturation in ambient air (96\%). Physical examination showed diminished respiration and moist rales in the left lower lung. The right thigh showed swelling, increased tension, elevated skin temperature, tenderness, limited movement but no redness. Oral examination showed several dental caries and calculus, but no obvious signs of gingivitis.

Laboratory results showed a high white blood cell count $\left[22.69 \times 10^{9} / \mathrm{L}\right.$; normal range, $\left.(3.5-9.5) \times 10^{9} / \mathrm{L}\right]$, a high neutrophil percentage (95\%), a normal hemoglobin count $(146 \mathrm{~g} / \mathrm{L})$, a high level of $\mathrm{N}$-terminal pro-brain natriuretic peptide (551 ng/L; normal range, 0-227 ng/L), a high D-dimer level $(1.22 \mathrm{mg} / \mathrm{L}$; normal range, $<0.55 \mathrm{mg} / \mathrm{L})$, a significantly high procalcitonin level $(2.16 \mathrm{ng} / \mathrm{L}$; normal range, $<0.046 \mathrm{ng} / \mathrm{L})$, a low blood albumin level $(22.9 \mathrm{~g} / \mathrm{L}$; normal range, $40-55 \mathrm{~g} / \mathrm{L}$ ), and a normal creatine kinase level (91 IU/L; normal range, 19-226 IU/L). Body temperature was monitored daily and white blood cell counts were measured every three days throughout hospitalization, until they were normal (Figure 1). Assays of liver and kidney function, coagulation function, immunological markers, and tumor markers were normal. The patient tested negative for SARS-CoV-2 based on PCR testing of a nasopharyngeal swab.

Transthoracic echocardiography showed mild enlargement of the left atrium, but no thrombus was detected using lower limb compression venous Doppler ultrasonography. Computed tomography of the chest showed varying degrees of expansion and plugging in the bronchus of the left lower lobe, with slight infiltration (Figure 2A). Enhanced computed tomography of the right thigh showed obvious soft tissue swelling and scattered effusion, as well as multiple soft tissue nodules and mass shadows around the right femur. The largest mass measured $8.2 \mathrm{~cm} \times 4.3 \mathrm{~cm}$. The edge of the lesion was fuzzy, and it did not show a clear boundary with adjacent muscle (Figure $2 B$ ).

Based on the underlying bronchiectasis and the possibility of suppurative infection of the right thigh, the patient was initially treated empirically with piperacillintazobactam $(4.5 \mathrm{~g}$ every $8 \mathrm{~h})$ and tinidazole $(0.8 \mathrm{~g}$ once a day) therapy from the first day after admission. After magnetic resonance imaging confirmed the formation of multiple abscesses in the patient's thigh (Figure 3), ultrasound-guided percutaneous catheter drainage was 

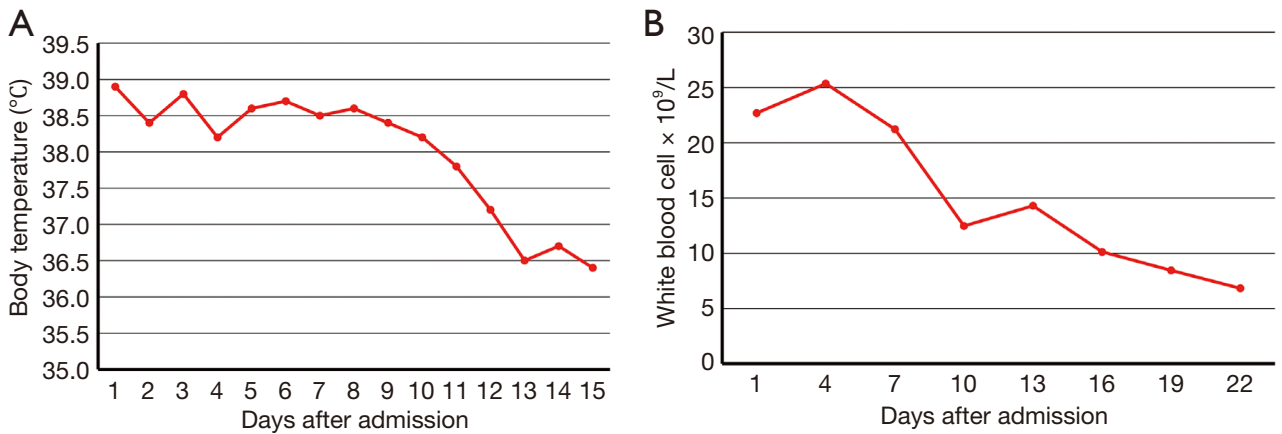

Figure 1 The curve of body temperature and white blood cell count during hospitalization. Antibacterial treatment with ceftriaxone and vancomycin were administered from day 7 after admission. (A) Body temperature; (B) white blood cell.
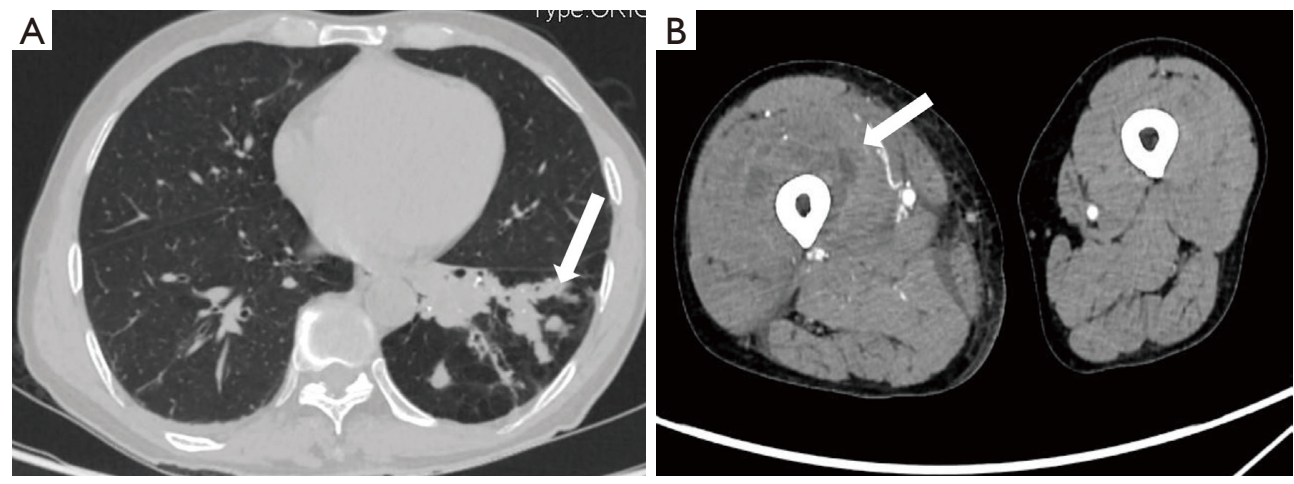

Figure 2 Chest and thigh computed tomography scan. (A) Computed tomography of the chest showing bronchiectasis with infection in the left lower lung (white arrow); (B) enhanced computed tomography of the right thigh showing obvious soft tissue swelling and multiple, low-density mass shadows (white arrow).
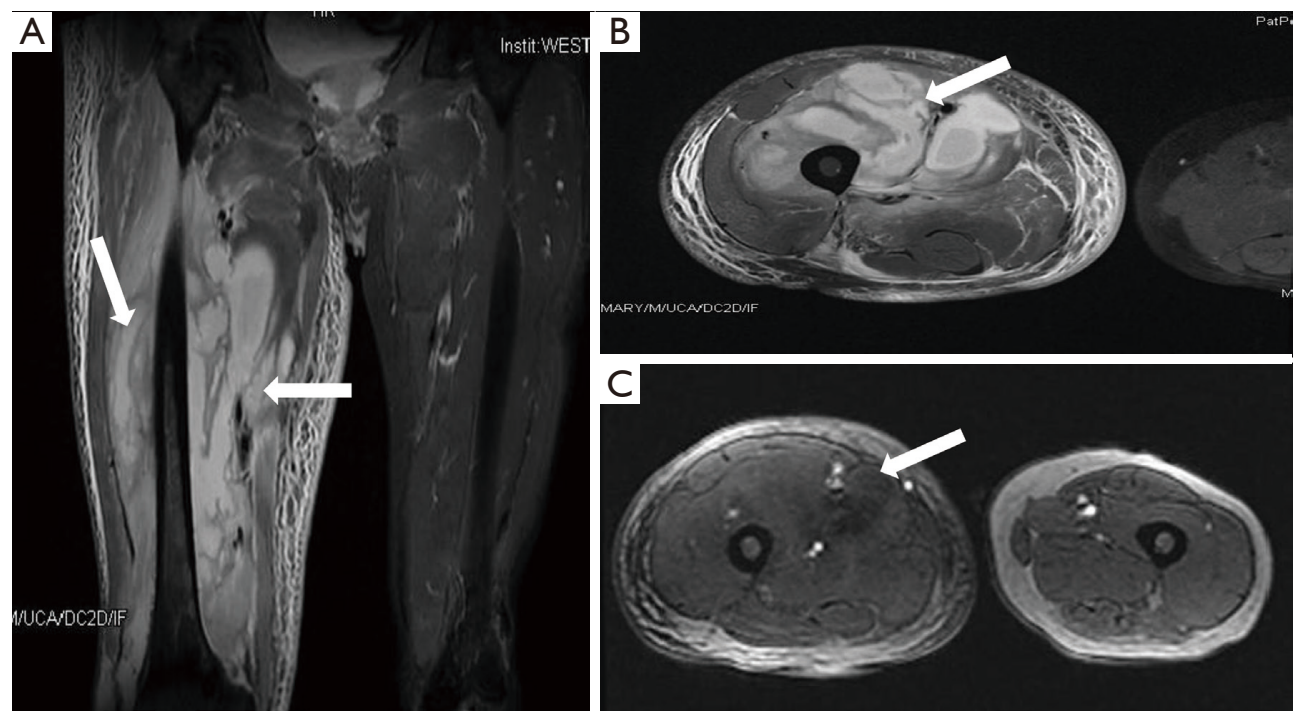

Figure 3 Thigh magnetic resonance images. (A) Axial and (B) coronal enhanced magnetic resonance images showing multiple abscesses in the right thigh (white arrows); (C) coronal unenhanced magnetic resonance images showing obvious swelling of the right thigh (white arrow). 
A
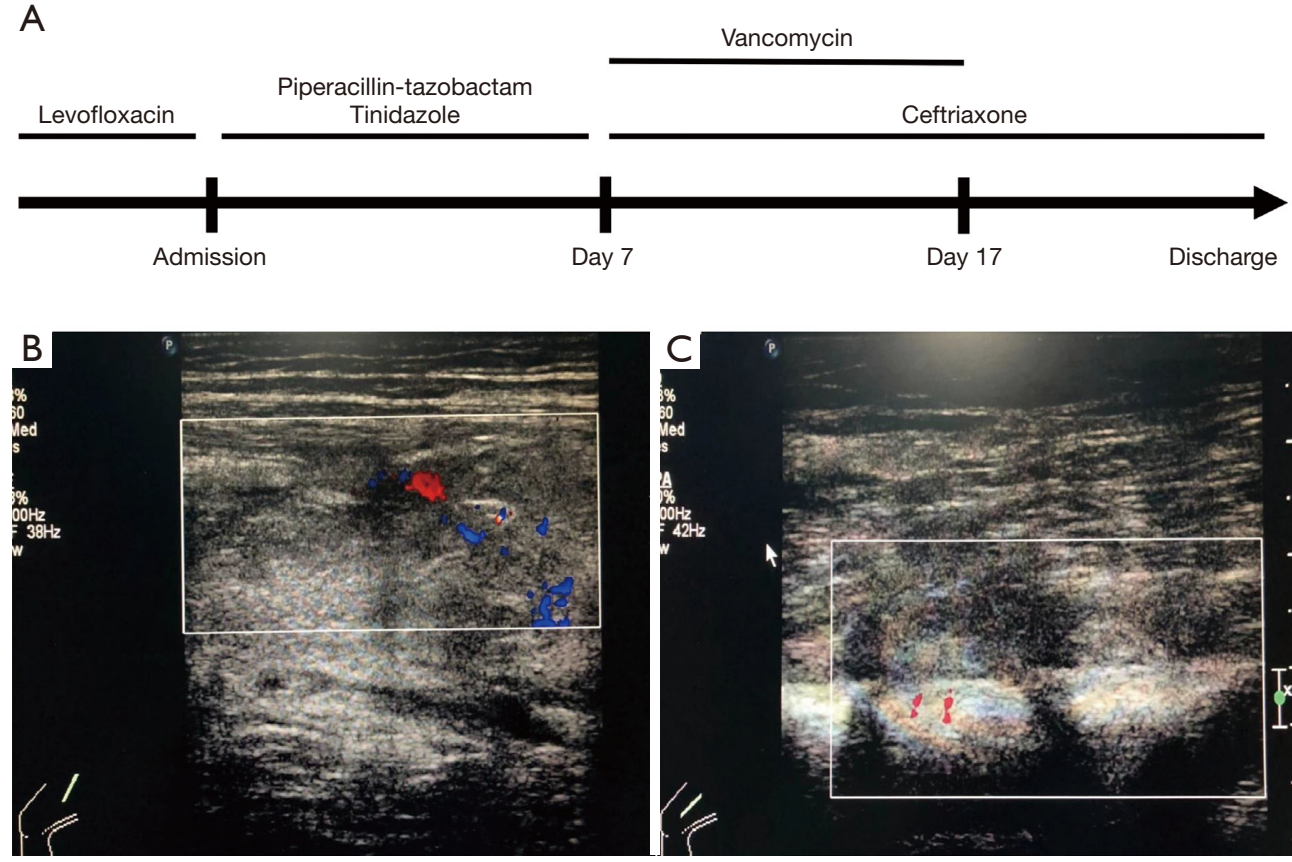

Figure 4 Antibiotic treatment timeline and the color ultrasonography soon before the discharge. (A) Timeline; (B,C) color ultrasonography showing significant absorption of several thigh abscesses.

performed, and about $500 \mathrm{~mL}$ of reddish pus was drained within the first $24 \mathrm{~h}$ of drainage.

Metagenomic next-generation sequencing of the pus sample on day 3 after admission detected the presence of oral anaerobic bacteria, including oral Prevotella and endodontic Porphyromanus. Culture tests of abscess fluid on days 3, 4 and 7 after admission indicated the growth of $S$. constellatus subsp. constellatus, whereas S. constellatus was not detected in culture tests of the patient's blood on days 1,3 and 7, and in sputum samples on days 1, 3, 7 and 9 after admission. The pathogen was identified by matrixassisted laser desorption/ionization time-of-flight mass spectrometry. Drug sensitivity tests of the abscess fluid cultures showed that the $S$. constellatus subsp. constellatus was sensitive to chloramphenicol, linezolid, cefotaxime, vancomycin and ceftriaxone, but resistant to penicillin $G$, erythromycin and clindamycin. Thus, the combination of piperacillin-tazobactam and tinidazole therapy was replaced with ceftriaxone ( $2 \mathrm{~g}$ once a day) and vancomycin $(0.5 \mathrm{~g}$ every 8 h) (Figure $4 A$ ).

By four days after this change in antibiotic therapy and eight days after drainage began, the patient's cough, expectoration, fever, and thigh pain showed significant improvement. Color ultrasonography soon before the patient's discharge showed substantial absorption of several thigh abscesses (Figure 4B,C). The patient was discharged after completing a 4-week course of antibiotic treatment. Follow-up at 3 months after discharge showed no recurrence of the thigh abscess.

All procedures performed in this study were in accordance with the ethical standards of the institutional and/or national research committee(s) and with the Helsinki Declaration (as revised in 2013). Written informed consent was obtained from the patient for publication of this case report and accompanying images. A copy of the written consent is available for review by the editorial office of this journal.

\section{Discussion}

In clinical practice, as deep tissue, thigh muscles are not uncommon sites of abscess formation. Abscesses occur primarily as a result of infection, but they can also arise through direct spread from adjacent infected lesions, or through hematogenous dissemination from other sources (11). Staphylococcus aureus and Escherichia coli are common pathogens that cause abscess formation, but abscesses can also be caused by less frequent pathogens such 
as Mycobacterium tuberculosis $(11,12)$. As far as we know, the present case is the first report of thigh abscesses caused by S. constellatus subsp. constellatus in a patient with exacerbation of bronchiectasis.

There is no clear understanding of the clinical burden of $S$. constellatus, due to a lack of epidemiological data. $S$. constellatus requires $5 \% \mathrm{CO}_{2}$ or an anaerobic environment to grow; therefore, it is easily missed by microbial culture teats conducted in aerobic environments (13). Poor communication between clinicians and laboratory technicians may also bias against its detection: $S$. constellatus observed in samples are often overlooked and erroneously considered as commensal contamination (1). In addition, this microbe is sensitive to most antibiotics, and overuse of antibiotics can lead to a negative culture test. In our patient, abscess fluid cultures consistently tested positive for $S$. constellatus subsp. constellatus due to the abscess fluid have higher bacterial load and was paid more attention to the detection of anaerobic bacteria.

Metagenomic next-generation sequencing can detect pathogens in a target-independent manner. It is gradually becoming an irreplaceable supplement to routine laboratory methods, helping make treatment more precise and reduce antibiotic abuse (14). However, it failed to definitively identify $S$. constellatus in our patient, although it did detect other oral pathogens in abscess fluid. The most likely explanations include the accuracy and completeness of the sequencing database in laboratory, and the false negative caused by human omission of the detected sequences that were obviously less than those of other aforementioned oral anaerobes. Thus, our case highlights the need to interpret the results of metagenomic next-generation sequencing with caution and to pay due attention to quality control, evaluation methods, and other factors (14).

The oral cavity is the main source of $S$. constellatus in the body (1). Previous case reports describing abscesses caused by $S$. constellatus mainly refer to odontogenic infections $(15,16)$. Abscesses caused by $S$. constellatus after surgery and trauma have also been rarely reported (17). S. constellatus, especially the $S$. constellatus subsp. constellatus, can cause abscesses and bacteremia, which may be related to the structure of polysaccharidic capsules on these bacteria, and the sagA homologue of the $S$. constellatus subsp. constellatus is associated with its cytotoxicity (2).

Although infections caused by $S$. constellatus can be observed in individuals with normal immunity, a growing list of conditions were identified as potential predisposing factors, such as alcohol abuse, trauma, surgery, immunosuppression, multimorbidity, rheumatic diseases, cardiovascular diseases, chronic obstructive pulmonary disease, concurrent infection, and diabetes mellitus $(3,18)$. Our patient had chronic underlying bronchiectasis and was suffering an acute exacerbation, so further work should examine whether this condition also is a predisposing factor of $S$. constellatus infection. In addition, the origin of the $S$. constellatus in the thigh abscess in our patient is unclear. It seems that poor oral hygiene was the most likely cause of bacteremia due to $S$. constellatus. However, the other possibility is the lung, even if sputum culture tests were negative, because primary lung infection can lead to pyopneumothorax and pulmonary abscess $(7,19,20)$. Indeed, this can further evidence by another study which showed the $S$. anginosus group can induce chronic pulmonary infections and was the numerically dominant pathogen at the onset of $39 \%$ of acute pulmonary exacerbations in cystic fibrosis patients (21). Those investigators recommended expanding S. anginosus group surveillance to bronchiectasis patients. Thus, we believe that $S$. constellatus, although it is uncommon, should still be considered as a potential pathogen to exacerbation of bronchiectasis, especially in patients with secondary abscesses in other tissues and organs. To further our understanding of $S$. constellatus, the link between it and bronchiectasis should be explored in future work.

S. constellatus is sensitive to a range of antibiotics, so its treatment is considered to be uncomplicated. However, suppurative infections at important sites, such as the central nervous system, are associated with a higher risk of death. Penicillins such as ampicillin are the first choice for the treatment of $S$. constellatus infections, followed by erythromycin, chloramphenicol, clindamycin, ofloxacin, vancomycin, cephalosporin, and other antibiotics (22). In one report, linezolid was used to treat pyopneumothorax caused by S. constellatus infection (18). In clinical practice, the above-mentioned drugs can be empirically used for initial treatment without obtaining results from appropriate drug sensitivity tests, but the local epidemiological situation should be taken into account. Even though $>90 \%$ of patients with $S$. constellatus infections can be successfully treated with $\beta$-lactam antibiotics, resistant strains have been reported and should be identified as quickly as possible (23). Drug therapy can be adjusted after conducting sensitivity tests. In the case of our patient, the repeated use of penicillin antibiotics for the treatment of bronchiectasis exacerbations may have led to insensitivity.

Although consensus or universal guidelines are lacking, 
the recommended general duration of antimicrobial treatment for $S$. constellatus is 4-6 weeks, especially when there is abscess formation (24). Timely and adequate drainage of pus is the cornerstone of treatment. Direct surgical incision or percutaneous catheters can be chosen for the drainage of pus, but the latter is more widely used due to its safety and high success rate $(>80 \%)$. Drainage can be performed under the guidance of ultrasonography or computed tomography (11). Our patient's symptoms improved after adequate antibiotic therapy, yet we attribute the remission more to active percutaneous catheter drainage guided by color ultrasonography.

As conclusions, here we report the first case of thigh abscess caused by $S$. constellatus subsp. constellatus in a patient suffering from bronchiectasis exacerbation. Further studies need to be conducted to understand the range of suppurative infections caused by $S$. constellatus, even though it is a rare pathogen. This will help in the timely identification of such infections in clinical practice. Although the prognosis of most patients with such infections is relatively good, the timely administration of sensitive antibiotics along with abscess drainage may prevent further deterioration and recurrence.

\section{Acknowledgments}

Funding: This work was supported by the Sichuan Science and Technology Project [2017SZ0068 to HM] and the Deyang Science and Technology Project [2018SZS056 to YH.F].

\section{Footnote}

Reporting Checklist: The authors have completed the CARE reporting checklist. Available at https://apm.amegroups. com/article/view/10.21037/apm-21-740/rc

Peer Review File: Available at https://apm.amegroups.com/ article/view/10.21037/apm-21-740/prf

Conflicts of Interest: All authors have completed the ICMJE uniform disclosure form (available at https://apm. amegroups.com/article/view/10.21037/apm-21-740/coif). The authors have no conflicts of interest to declare.

Ethical Statement: The authors are accountable for all aspects of the work in ensuring that questions related to the accuracy or integrity of any part of the work are appropriately investigated and resolved. All procedures performed in this study were in accordance with the ethical standards of the institutional and/or national research committee(s) and with the Helsinki Declaration (as revised in 2013). Written informed consent was obtained from the patient for publication of this case report and accompanying images. A copy of the written consent is available for review by the editorial office of this journal.

Open Access Statement: This is an Open Access article distributed in accordance with the Creative Commons Attribution-NonCommercial-NoDerivs 4.0 International License (CC BY-NC-ND 4.0), which permits the noncommercial replication and distribution of the article with the strict proviso that no changes or edits are made and the original work is properly cited (including links to both the formal publication through the relevant DOI and the license). See: https://creativecommons.org/licenses/by-nc-nd/4.0/.

\section{References}

1. Asam D, Spellerberg B. Molecular pathogenicity of Streptococcus anginosus. Mol Oral Microbiol 2014;29:145-55.

2. Tabata A, Sato Y, Maya K, et al. A streptolysin S homologue is essential for $\beta$-haemolytic Streptococcus constellatus subsp. constellatus cytotoxicity. Microbiology (Reading) 2014;160:980-91.

3. Potsios C, Xaplanteri P, Zoitopoulos V, et al. Pyogenic Spondylodiscitis due to Streptococcus constellatus in an Immunocompromised Male Patient: A Case Report and Review of the Literature. Case Rep Infect Dis 2019;2019:9364951.

4. Khan MZ, Tahir D, Kichloo A, et al. Pyogenic Liver Abscess and Sepsis Caused by Streptococcus constellatus in the Immunocompetent Host. Cureus 2020;12:e9802.

5. Ye RH, Yang JC, Hong HH, et al. Descending necrotizing mediastinitis caused by Streptococcus constellatus in an immunocompetent patient: case report and review of the literature. BMC Pulm Med 2020;20:43.

6. Hammond RF, Jasionowska S, Awad WI. Aortic valve replacement with sutureless Perceval S valve: A case report of aortic root homograft failure in the setting of Streptococcus constellatus endocarditis. J Card Surg 2020;35:2829-31.

7. Elhussein TA, Hutchison SJ. Streptococcus constellatus community acquired pneumonia with subsequent isolated 
pulmonic valve endocarditis and abscess formation in a structurally normal heart. J Cardiovasc Ultrasound 2014;22:91-4.

8. Akashi M, Tanaka K, Kusumoto J, et al. Brain Abscess Potentially Resulting from Odontogenic Focus: Report of Three Cases and a Literature Review. J Maxillofac Oral Surg 2017;16:58-64.

9. Mills D, Sharon B, Schneider K. Streptococcus constellatus Tubo-ovarian Abscess in a Non-Sexually Active Adolescent Female. Pediatr Emerg Care 2018;34:e100-e101.

10. Palomino-Nicás J, González E, Arroyo A, et al. Pyomyositis due to Eubacterium lentum and Streptococcus constellatus from a periodontal source. Clin Infect Dis 1996;22:176-8.

11. Abbas TO. Pelvic primary staphylococcal infection presenting as a thigh abscess. Case Rep Surg 2013;2013:539737.

12. Dharmapalan A, Vijaykumar R, Bhoopal S. Renal tuberculosis presenting as thigh abscess. Indian J Surg 2013;75:446-8.

13. Grinwis ME, Sibley CD, Parkins MD, et al. Characterization of Streptococcus milleri group isolates from expectorated sputum of adult patients with cystic fibrosis. J Clin Microbiol 2010;48:395-401.

14. Zhao Y, Chen J, Bai B, et al. Pathogen determination from clinical abscess fluids using metagenomic next-generation sequencing. Folia Microbiol (Praha) 2021;66:197-202.

15. Al Asaadi Z, Srinivasan B, Melchers LJ, et al. Streptococcus constellatus causing bony destruction secondary to odontogenic infection: three rare cases. Br J Oral Maxillofac Surg 2019;57:594-6.

16. Li E, Distefano A, Sohrab M. Necrotizing Orbital Cellulitis Secondary to Odontogenic Streptococcus constellatus. Ophthalmic Plast Reconstr Surg

Cite this article as: Feng Y, Wang Y, Zeng C, Mao H. Pyogenic thigh abscess caused by Streptococcus constellatus subsp. constellatus in a patient with exacerbation of bronchiectasis: a case report. Ann Palliat Med 2022;11(4):1526-1532. doi: 10.21037/ apm-21-740 2018;34:e160-e162.

17. Tymon-Rosario J, Atrio JM, Yoon HA, et al. Streptococcus constellatus Peritonitis and Subsequent Septic Shock following Intrauterine Device Removal. Case Rep Obstet Gynecol 2019;2019:6491617.

18. Zhang Z, Xiao B, Liang Z. Successful treatment of pyopneumothorax secondary to Streptococcus constellatus infection with linezolid: a case report and review of the literature. J Med Case Rep 2020;14:180.

19. Che Rahim MJ, Mohammad N, Wan Ghazali WS. Pyopneumothorax secondary to Streptococcus milleri infection. BMJ Case Rep 2016;2016: bcr2016217537.

20. Porta G, Rodríguez-Carballeira M, Gómez L, et al. Thoracic infection caused by Streptococcus milleri. Eur Respir J 1998;12:357-62.

21. Sibley CD, Parkins MD, Rabin HR, et al. A polymicrobial perspective of pulmonary infections exposes an enigmatic pathogen in cystic fibrosis patients. Proc Natl Acad Sci U S A 2008;105:15070-5.

22. Rams TE, Feik D, Mortensen JE, et al. Antibiotic susceptibility of periodontal Streptococcus constellatus and Streptococcus intermedius clinical isolates. J Periodontol 2014;85:1792-8.

23. Giuliano S, Rubini G, Conte A, et al. Streptococcus anginosus group disseminated infection: case report and review of literature. Infez Med 2012;20:145-54. Corrected in Infez Med 2012;20:316.

24. Mohanty S, Panigrahi MK, Turuk J, et al. Liver Abscess due to Streptococcus constellatus in an Immunocompetent Adult: A Less Known Entity. J Natl Med Assoc 2018;110:591-5. 